Marquette University

e-Publications@Marquette

Mechanical Engineering Faculty Research and

Publications

Mechanical Engineering, Department of

$10-2016$

\title{
A Comparison of Specularly Reflective Boundary Conditions and Rotationally Invariant Formulations for Discrete Ordinate Methods in Axisymmetric Geometries
}

Jian Cai

University of Wyomming

Somesh Roy

Marquette University, somesh.roy@marquette.edu

Michael F. Modest

University of California, Merced

Follow this and additional works at: https://epublications.marquette.edu/mechengin_fac

Part of the Mechanical Engineering Commons

\section{Recommended Citation}

Cai, Jian; Roy, Somesh; and Modest, Michael F., "A Comparison of Specularly Reflective Boundary Conditions and Rotationally Invariant Formulations for Discrete Ordinate Methods in Axisymmetric Geometries" (2016). Mechanical Engineering Faculty Research and Publications. 97.

https://epublications.marquette.edu/mechengin_fac/97 
Marquette University

e-Publications@Marquette

\section{Mechanical Engineering Faculty Research and Publications/College of Engineering}

This paper is NOT THE PUBLISHED VERSION.

Access the published version at the link in the citation below.

Journal of Quantitative Spectroscopy and Radiative Transfer, Vol. 182 (October 2016): 75-86. DOI. This article is (C) Elsevier and permission has been granted for this version to appear in e-

Publications@Marquette. Elsevier does not grant permission for this article to be further copied/distributed or hosted elsewhere without the express permission from Elsevier.

\section{A Comparison of Specularly Reflective Boundary Conditions and Rotationally Invariant Formulations for Discrete Ordinate Methods in Axisymmetric Geometries}

\section{Jian Cai}

Department of Mechanical Engineering, University of Wyoming, Laramie, WY

Somesh Roy

School of Engineering, University of California, Merced, CA

Michael F. Modest

School of Engineering, University of California, Merced, CA

\section{Abstract}

In simulations of periodic or symmetric geometries, computational domains are reduced by imaginary boundaries that exploit the symmetry conditions. Two boundary conditions are proposed for Discrete Ordinate Methods to solve axisymmetric radiation problems. Firstly, a specularly reflective boundary 
condition similar to that is used in Photon Monte Carlo methods is developed for Discrete Ordinate Methods. Secondly, the rotational invariant formulation is revisited for axisymmetric wedge geometries. Correspondingly, a new rotationally invariant boundary condition specially designed for axisymmetric problems on wedge shape is proposed to enforce the rotational invariance properties possessed by the radiative transfer equation (RTE) but violated by three-dimensional conventional Discrete Ordinate Methods. Both boundary conditions have the advantage that the discretization and linear equation solution procedures of conventional three-dimensional DOM are not affected by changing to a reduced geometry. Consistency, accuracy and efficiency of the new boundary conditions are demonstrated by multiple numerical examples involving periodic symmetry and axisymmetry. $A$ comparison between specularly reflective boundary conditions and the rotationally invariant formulation shows that the latter offers several advantages for wedge geometries. In other symmetry conditions, when the rotational invariant formulation is not applicable, specular reflective boundary conditions are still effective.

\section{Keywords}

Thermal radiation, Nongray radiation, Discrete Ordinate Methods, Axisymmetric enclosures

\section{Introduction}

Periodic and symmetric geometries are frequently encountered in engineering applications. Examples of such geometries include periodic structures, plane symmetry and axisymmetry. In the simulations of these problems, flow variables are solved for only a section of the domain. The computational domain is separated from the rest by imaginary boundaries, upon which symmetric constraints as opposed to physical conditions are applied.

While symmetry constraints may be easily expressed into mathematical formulas for scalar and vector fields as frequently performed in CFD simulations, they present challenges for radiative intensities. This is because the radiative intensities are functions of both spatial and directional coordinates [1]. Plane and rotational symmetric conditions are complicated by the additional directional variations. As an example, scalar flow variables in an axisymmetric problem are only functions of radial and axial locations. In flow solvers designed for three-dimensional coordinates only, a wedge geometry that has only one layer of cells in the azimuthal direction may be employed. Symmetry conditions are imposed on the imaginary wedge planes. However, even in these axisymmetric problems, the radiative intensities are three dimensional, i.e., light propagation is not confined to the plane formed by the radial and axial direction vectors. Such issues are also observed in Photon Monte Carlo (PMC) methods for radiative transfer. In PMC methods, the wedge planes may be treated as specularly reflective boundaries, i.e., a ray hitting the wedge plane is reflected back into the computational domain like hitting a perfect mirror.

When applying Discrete Ordinate Methods [1], [2] to axisymmetric simulations, three methods are typically employed. In the first method, the radiative transfer equation (RTE) is solved for ordinate intensities in a full three-dimensional axisymmetric domain, as in Refs. [3], [4], [5]. This method cannot take advantage of symmetry to reduce computational cost. In the second method, the RTE is formulated in cylindrical coordinates directly as in Refs. [6], [7], [8], [9], [10], [11]. However, this approach requires dedicated implementations of DOM equations for two-dimensional problems. In the 
third method, a reduced mesh based on symmetry is used to discretize the RTE formulated for Cartesian coordinates [12], [13]. Axisymmetry is enforced by special treatment of the wedge boundaries. This method simplifies the implementation of the RTE, because the same equations, discretization and linear equation solution methods are used for both reduced and full geometries. However, the resulting new wedge boundaries require different treatment from that is employed for scalar or vector partial differential equations.

In this work, after a short review of the Discrete Ordinate Methods and their Finite Volume discretizations in 2.1 Discrete ordinate methods, 2.2 Finite volume discretization, a specularly reflective boundary condition is proposed in Section 2.3 for Discrete Ordinate Methods in recognition of the fact that the ordinates have similarities to rays in Photon Monte Carlo methods. The specularly reflective boundary conditions are expressed as mathematical boundary conditions for the first-order partial differential equations that govern spatial variations of each ordinate depending on the spatial relationship between the surface normal and the ordinate directions. It is shown that the implementation of the specularly reflective boundary condition varies with the finite volume interpolation scheme for the face values. The specularly reflective boundary condition can be applied to both periodic and axisymmetric reduced geometries. In Section 2.4, a rotationally invariant formulation is derived for axisymmetric problems in wedge geometries. Its implementation results in a specially designed boundary condition for the wedge boundaries (Section 2.5), so that the remaining Finite Volume discretization of the DOM equations is unchanged. Similarities and differences of the two boundary conditions are highlighted in Section 2.6. Several tests are performed in Section 3 to further examine the consistency, accuracy and efficiency of the two boundary conditions in reduced geometries.

\section{Theoretical background}

\subsection{Discrete ordinate methods}

In this section, the Discrete Ordinate Methods for solving the radiative transfer equation are briefly reviewed. The presentation only focuses on necessary content for the new development as opposed to completeness. We will limit the theoretical discussion to non-scattering participating media, because the treatment of scattering is not essential to this work and does not impose new technical difficulties. Readers are referred to Ref. [1] for more comprehensive discussions of the DOM and Ref. [2] for the review of recent developments.

The radiative transfer equation (RTE) for a radiatively participating gray medium with emission and absorption is a first order differential equation:

$$
\hat{s} \cdot \nabla_{x} I(\boldsymbol{x}, \hat{s})=\kappa I_{\mathrm{b}}(\boldsymbol{x})-\kappa I(\boldsymbol{x}, \hat{\boldsymbol{s}})
$$

where $\mathrm{x}$ is the spatial coordinate, $\hat{s}$ a unit direction vector, $\kappa$ the absorption coefficient, $I$ the radiative intensity and $I_{\mathrm{b}}$ the blackbody intensity (or Planck function). The subscript $x$ on the gradient operator $\nabla$ emphasizes that the gradient is with respect to spatial coordinates only. 
In Discrete Ordinate Methods the directional variation of the radiative intensity is expressed by intensities on a set of prescribed directions, known as the ordinates. For each of

the $n$ ordinates $\left(\hat{\boldsymbol{s}}_{i}, i=1, \ldots, n\right)$, the corresponding intensity $\left(I_{i}=I\left(\boldsymbol{x}, \hat{\boldsymbol{s}}_{i}\right)\right)$ is determined by solving the RTE for direction $\hat{s}_{i}$, i.e.,

(2)

$$
\hat{s}_{i} \cdot \nabla I_{i}(\boldsymbol{x})=\kappa I_{\mathrm{b}}(\boldsymbol{x})-\kappa I_{i}(\boldsymbol{x})
$$

The resulting DOM RTE is a first order partial differential equation and depends on spatial coordinates only. Each ordinate $\hat{\boldsymbol{S}}_{i}$ has a directional quadrature weight $w_{i}$ such that a directional integral is converted into a sum over quadratures. In particular,

(3)

$$
\int d \Omega=\sum_{i} w_{i}=4 \pi
$$

(4)

$$
\int I d \Omega=\sum_{i} I_{i} w_{i}=G
$$

where $G$ is the incident radiation.

\subsection{Finite volume discretization}

Eq. (2) may be solved numerically by Finite Volume Methods, i.e., Eq. (2) is integrated over a cell volume before the Gaussian theorem is used to convert differential operations into algebraic operations. For example, consider a cell $C$ with a volume $V_{c}$ enclosed by $n_{f}$ faces (Fig. 1). The RTE is integrated over this cell according to

$$
\int_{V_{c}} \hat{\boldsymbol{s}}_{i} \cdot \nabla I_{i}(\boldsymbol{x}) d V=\int_{V_{c}} \kappa I_{\mathrm{b}}(\boldsymbol{x}) d V-\int_{V_{c}} \kappa I_{i}(\boldsymbol{x}) d V
$$

For the right-hand side, the cell-center values of radiative intensity $\left(I_{i c}\right)$ and properties $\left(\kappa_{c}\right)$ are defined such that

(6)

$$
\int_{V_{c}} \kappa I_{\mathrm{b}}(\boldsymbol{x}) d V-\int_{V_{c}} \kappa I_{i}(\boldsymbol{x}) d V=V_{c} \kappa_{c}\left(I_{b c}-I_{i c}\right)
$$


where $I_{b c}$ is the Planck function evaluated at the cell-valued thermodynamic state. The left hand side is related to $I_{i}$ at the faces employing the Gaussian theorem

(7)

$$
\int_{V_{c}} \hat{\boldsymbol{s}}_{i} \cdot \nabla I_{i}(\boldsymbol{x}) d V=\int_{S_{c}} I_{i}(\boldsymbol{x}) \hat{\boldsymbol{s}_{i}} \cdot d \boldsymbol{S}=\sum_{f} \int_{S_{f}} I_{i}(\boldsymbol{x}) \hat{\boldsymbol{s}_{i}} \cdot d \boldsymbol{S}=I_{i f} \hat{\boldsymbol{S}_{i}} \cdot \boldsymbol{S}_{f}
$$

where $I_{i f}$ is the intensity $I_{i}$ at face $f$, and $\boldsymbol{S}_{f}$ the vector surface area of face $f$.

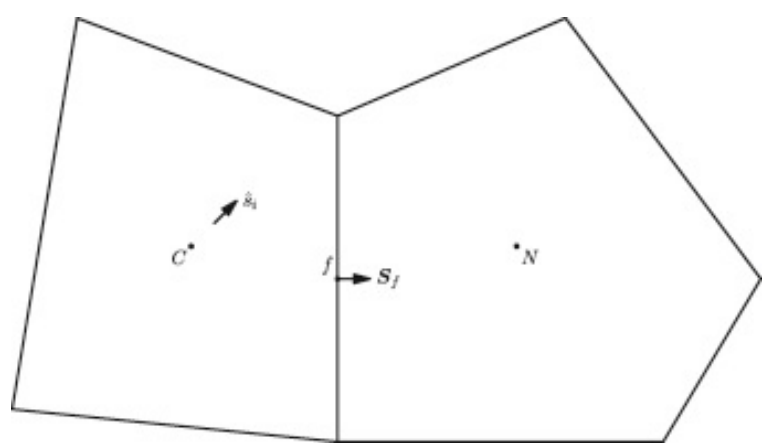

Fig. 1. Geometry for convection schemes.

Further development is now required to express the face values $I_{i f}$ in terms of $I_{i}$. When the face $f$ is an internal (non-boundary) face, $I_{i f}$ is usually expressed as a function of $I_{i}$ in the current cell $\left(I_{i C}\right)$ and that in the neighbor cell $\left(I_{i N}\right)$ that shares face $f$

(8)

$$
I_{i f}=F\left(I_{i C}, I_{i N}, \hat{s}_{i}, \boldsymbol{S}_{f}\right)
$$

The simplest scheme is the step scheme known as the upwind scheme, i.e.,

(9)

$$
I_{i f}=F\left(I_{i C}, I_{i N}, \hat{\boldsymbol{s}_{i}}, \boldsymbol{S}_{f}\right)= \begin{cases}I_{i C}, & \hat{\text { if }} \boldsymbol{s}_{i} \cdot \boldsymbol{S}_{f} \geq 0 \\ I_{i N}, & \text { otherwise }\end{cases}
$$

However, when face $f$ is on a computational boundary, a boundary condition is needed to determine the value of $I_{i}$ on an ordinate $s_{i}$ outgoing from the face. For physical boundaries with prescribed radiative properties, the intensity from the boundary is calculated from temperature, emissivity and incoming intensities. For symmetry boundaries, a specularly reflective boundary condition is commonly used in the practice of Photon Monte Carlo methods.

\subsection{Specularly reflective boundary condition}

Light is reflected on a specularly reflective boundary as if on a mirror, as shown in Fig. 2 . The top half is within the computational domain, and is separated from the imaginary domain by a specularly 
reflective boundary $\left(O O^{\prime}\right)$. The reflected direction $\left(\boldsymbol{s}_{r}\right)$ of an incoming direction $\left(\boldsymbol{s}_{i}\right)$ after hitting a face with a normal direction $\boldsymbol{S}_{f}$ is

$$
\widehat{\boldsymbol{s}}_{r}=\widehat{\boldsymbol{s}}_{i}-2\left(\frac{\boldsymbol{S}_{f}}{\left\|\boldsymbol{S}_{f}\right\|} \cdot \widehat{\boldsymbol{s}}_{i}\right) \frac{\boldsymbol{S}_{f}}{\left\|\boldsymbol{S}_{f}\right\|}
$$

Because of the reflective symmetry imposed by boundary, the intensities of the boundary cell $(C)$ and its image $\left(C^{\prime}\right)$ are related by

$$
I_{i^{\prime} C^{\prime}}=I_{i C}
$$

and the geometric constraints $\hat{\boldsymbol{s}}_{i}^{\prime}=\hat{\boldsymbol{s}}_{r}$.

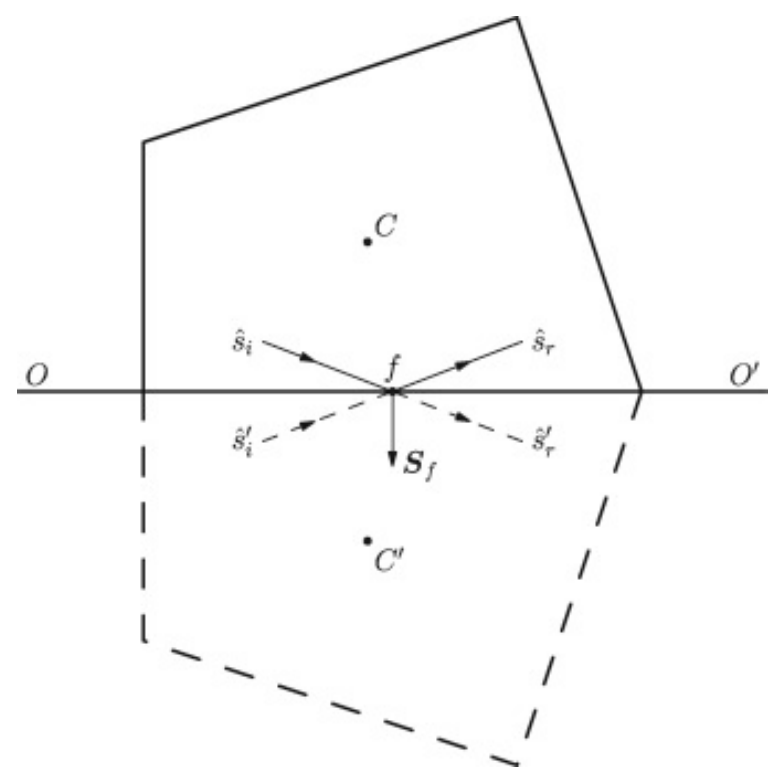

Fig. 2. Reflective boundary condition. The boundary $O O^{\prime}$ separates computational domain (shown above in solid lines) from its image (shown below in dashed lines). The cell centers are $C$ and its image $C^{\prime}$. An incoming ordinate $\left(\hat{\boldsymbol{s}}_{i}\right)$ is reflected back to computational domain as $\hat{\boldsymbol{s}}_{r} . \hat{\boldsymbol{s}}_{i}^{\prime}$ and $\hat{\boldsymbol{s}}_{r}^{\prime}$ are the images of the ordinates $\left(\hat{\boldsymbol{s}}_{i}\right)$ and $\hat{\boldsymbol{s}}_{r}$, correspondingly.

A successful reflective boundary condition should reproduce the same surface value as the internal surface when the full geometry including the image is employed. In other words, this requires that for any outgoing ordinate $\hat{\boldsymbol{s}}_{r}$, its intensity on the surface reads according to Eq. (8)

$$
I_{r f}=F\left(I_{r C}, I_{i^{\prime} C^{\prime}}, \hat{\boldsymbol{s}}_{r}, \boldsymbol{S}_{f}\right)=F\left(I_{r C}, I_{i C}, \hat{\boldsymbol{s}}_{r}, \boldsymbol{S}_{f}\right)
$$


Note that Eq. (11) is employed in the second equal sign to replace the cell-valued intensity in imaginary cells $\left(I_{i^{\prime} C^{\prime}}\right)$ with the equivalent intensity in boundary cells $\left(I_{r C}\right)$.

Similarly, the equation for the boundary conditions of the incoming ordinate $\hat{\boldsymbol{s}}_{i}$ can be derived,

$$
I_{i f}=F\left(I_{i C}, I_{r^{\prime} C^{\prime}}, \hat{\boldsymbol{s}}_{i}, \boldsymbol{S}_{f}\right)=F\left(I_{i C}, I_{r C}, \hat{\boldsymbol{s}}_{i}, \boldsymbol{S}_{f}\right)
$$

When the simplest step (upwind) scheme, Eq. (9), is used, the above boundary conditions can be simplified to

$$
I_{r f}=I_{i f}=I_{i C}
$$

This equation suggests that when the step scheme is used, the boundary condition for incoming ordinates is zero gradient, while the boundary condition for outgoing ordinate has a fixed value as the cell value from the incoming ordinate before reflection. These simplified conditions for the step scheme were also mentioned in Ref. [13]. However, for higher order spatial schemes, the boundary conditions are more complicated and involve cell values of the intensity of both incoming and outgoing ordinates in general, as shown in Eqs. (12), (13), respectively.

\subsection{Rotationally invariant formulation}

The RTE, Eq. (1), is rotationally invariant in axisymmetric problems, because the intensity depends simultaneously on both spatial and directional coordinates. As shown in Fig. 3, as the location rotates along the spatial azimuthal angle in a cylindrical coordinate, the radiative intensity rotates with the directional azimuthal angle in the same manner.

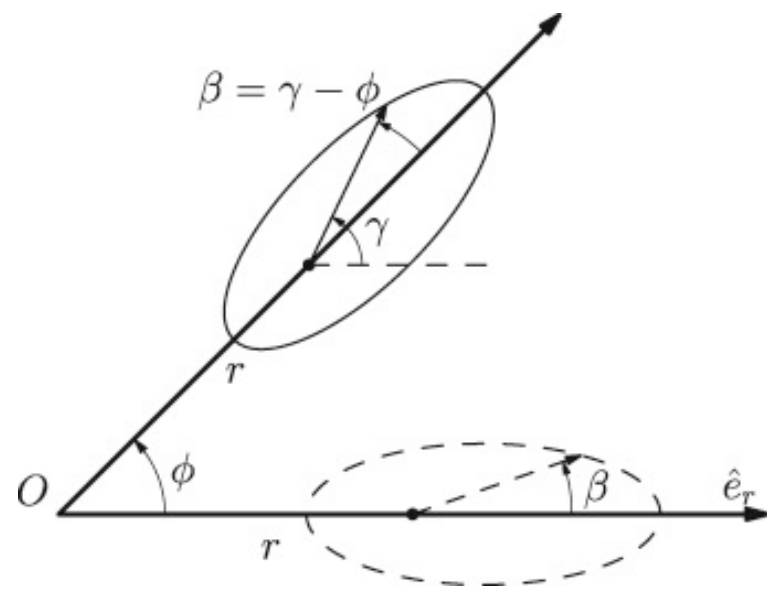

Fig. 3. Rotational invariance of radiative intensity in axisymmetric problems. Ellipses show intensity magnitude along different directions. The intensity value is invariant if both azimuthal angles change by the same amount.

However, after fixing the ordinates, the resulting DOM RTE, Eq. (2), is no longer rotationally invariant, because the scalar intensity [as opposed to the directional intensity in Eq. (1)] for any specific ordinate has azimuthal variations unless the ordinate is along the axial direction. This is different from the 
solution of any rotationally invariant scalar equation for axisymmetric problems in a cylindrical coordinate system, because the radiative intensity, which has five dimensions in three-dimensional space, still has four dimensions in axisymmetric problems.

To explicitly use the rotational invariance in an axisymmetric problem, we consider a radiative intensity of five dimensions, among which three are from cylindrical spatial coordinates $(r, z, \phi$ for radial location, axial location and azimuthal angle) and two are directional $(\theta, \beta$ for polar and azimuthal angles). The two azimuthal angles have separate origins, i.e., one is a spatial coordinate, while the other one is a directional coordinate. In an axisymmetric problem only four of them are independent, so that the intensity at other spatial azimuthal angles $\phi$ can be obtained by rotating the intensity at zero spatial azimuthal angle $(\phi=0)$ as shown in Fig. 3, i.e.,

$$
I(r, z, \phi ; \theta, \gamma)=I(r, z, 0 ; \theta, \gamma-\phi)
$$

This defines a rotationally invariant intensity $(I)$ of four independent dimensions normalized to the solution of the intensity at zero spatial azimuthal angle, i.e.,

$$
\hat{I}(r, z ; \theta, \beta)=I(r, z, \phi=0 ; \theta, \beta),
$$

and intensity at arbitrary spatial and directional azimuthal angles ( $\phi$ and $\gamma$, respectively) is related to the rotationally invariant intensity by

$$
I(r, z, \phi ; \theta, \gamma)=\hat{I}(r, z ; \theta, \gamma-\phi)
$$

To derive the corresponding radiative transfer equation for the rotationally invariant intensity $(I)$, we consider the change of five dimensional intensity $I(r, z, \phi ; \theta, \beta)$ traversing a length $\Delta s$ along its propagation direction $\hat{\boldsymbol{s}}=(\sin \theta \cos \beta, \sin \theta \sin \beta, \cos \theta)^{T}$. The projection of this small displacement to the axial direction $(z)$ is $\Delta z=\Delta s \cos \theta$. The projection to the $r-\phi$ plane, $\Delta s \sin \theta$, leads to a change of the $r$-coordinate $\Delta r=\Delta s \sin \theta \cos \beta$ and a change of the $\phi$-coordinate $\Delta \phi=\Delta s \sin \theta \sin \beta / r$, as shown in Fig. 4. 


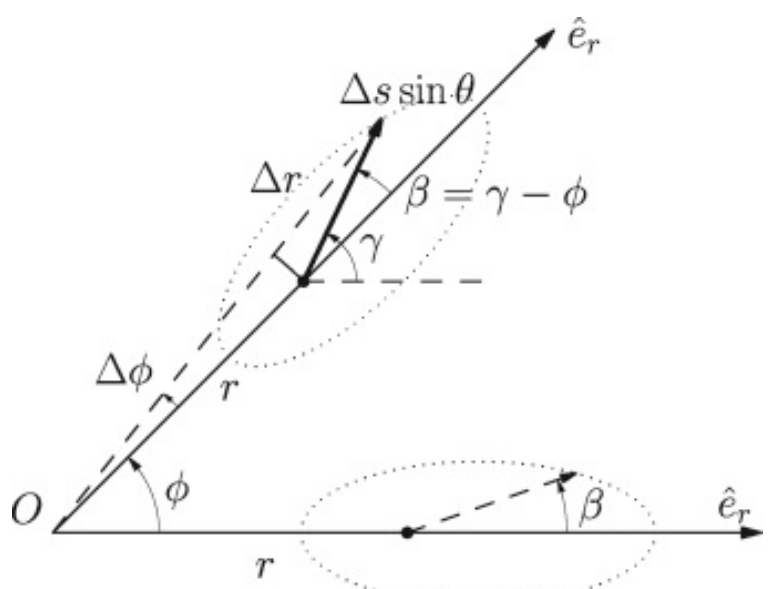

Fig. 4. Geometric decomposition of a small displacement in $r-\phi$ plane.

The change of intensity along the direction of propagation to the first order therefore is

$$
\Delta I=I(r+\Delta r, z+\Delta z, \phi+\Delta \phi ; \theta, \gamma)-I(r, z, \phi ; \theta, \gamma)
$$

$$
\Delta I=\hat{I}(r+\Delta r, z+\Delta z ; \theta, \beta-\Delta \phi)-\hat{I}(r, z ; \theta, \beta)
$$

(20)

$$
\Delta I=\frac{\partial \hat{I}}{\partial r} \Delta r+\frac{\partial \hat{I}}{\partial z} \Delta z-\frac{\hat{\partial I}}{\partial \beta} \Delta \phi
$$

$$
\Delta I=\Delta s\left\{\frac{\partial \hat{I}}{\partial r} \sin \theta \cos \beta+\frac{\partial \hat{I}}{\partial z} \cos \theta-\frac{\hat{\partial I}}{\partial \beta} \frac{\sin \theta \sin \beta}{r}\right\}
$$

Here $\beta=\gamma-\phi$ is used. On the other hand, the intensity is attenuated by absorption and augmented by emission according to

$$
\frac{\Delta I}{\Delta s}=\kappa I_{\mathrm{b}}-\kappa I=\hat{I_{\mathrm{b}}}-\kappa \hat{I}
$$

where $\hat{I}_{\mathrm{b}}(r, z ; \theta, \beta)=I_{\mathrm{b}}(r, z)$ is the isotropic blackbody emission. Combining Eqs. (21), (22), the radiative transfer equation for the rotationally invariant intensity $\hat{I}$ is 


$$
\sin \theta \cos \beta \frac{\partial \hat{I}}{\partial r}+\cos \theta \frac{\partial \hat{I}}{\partial z}-\frac{\sin \theta \sin \beta}{r} \frac{\partial \hat{I}}{\partial \beta}=\kappa \hat{I_{\mathrm{b}}}-\kappa \hat{I}
$$

The azimuthal angle $(\beta)$ here is inherited from the directional coordinate as opposed to the spatial coordinate. Furthermore, the negative sign before the derivative with respect to $\beta$ emphasizes that this derivative term is not the azimuthal component of the gradient operator $\hat{\boldsymbol{s}} \cdot \nabla I$ expressed in cylindrical coordinates, which would have a positive sign. Last but not least, the derivation here is based on rotational invariance, assuring that the rotational invariance properties of the original RTE [Eq. (1)], is strictly enforced in Eq. (23).

\subsection{Finite volume discretization in wedge geometry}

As shown in the previous section, the azimuthal angle $\beta$ is different from the azimuthal angle $\phi$ of a cylindrical coordinate system. Therefore, it requires special treatment before being incorporated into a Finite Volume Method solver. Two methods have been examined. The first method is to treat the derivative $\partial \hat{I} / \partial \beta$ or $(\sin \theta \sin \beta / r) \partial \hat{I} / \partial \beta$ as a new scalar variable, which acts as a source and is evaluated separately using numerical directional derivatives. However, the method was found numerically unstable. The second method, described in what follows, is based on a special interpretation of the angle $\beta$ through a new boundary condition, named rotationally invariant boundary condition hereafter.

After introducing a directional quadrature scheme for the $i$ th ordinate of intensity $I_{i}$ and directional coordinate $\left(\theta_{i}, \beta_{i}\right)$, the rotationally invariant RTE becomes

$$
\sin \theta_{i} \cos \beta_{i} \frac{\partial \hat{I_{i}}}{\partial r}+\cos \theta_{i} \frac{\partial \hat{I_{i}}}{\partial z}-\frac{\sin \theta_{i} \sin \beta_{i}}{r} \frac{\partial \hat{I}_{i}}{\partial \beta_{i}}=\kappa \hat{I_{\mathrm{b}}}-\kappa \hat{I}_{i}
$$

If we compare the rotational invariant RTE [Eq. (23)] with the original three-dimensional DOM RTE [Eq. (2)] for the ith ordinate expressed in cylindrical coordinates

$$
\hat{\boldsymbol{s}}_{i} \cdot \nabla I_{i}=\sin \theta_{i} \cos \beta_{i} \frac{\partial I}{\partial r}+\cos \theta_{i} \frac{\partial I}{\partial z}+\frac{\sin \theta_{i} \sin \beta_{i}}{r} \frac{\partial I}{\partial \phi}=\kappa I_{\mathrm{b}}-\kappa I,
$$

the two equations have the same form after assigning $d \phi=-d \beta$. This assignment is different from requiring $\phi=-\beta$ because when $d \phi=d \beta=0$ at the central plane of the wedge, $\beta=\beta_{i}$ and $\phi=0$. This suggests that Eq. (24) can be discretized in the same way as Eq. (25) except that the values at the two wedge boundaries should be swapped for the extra minus sign. 
In order to determine the face values at wedge boundaries, consider a Finite Volume discretization for axisymmetric problems using a wedge geometry with a wedge opening angle $\Delta \omega$ shown as in Fig. 5. For simplicity, we only focus on the discretization in the $r-\phi$ plane, because the discretization along the axial direction (z-coordinate) is identical to that in Cartesian coordinates. The Finite Volume Discretization of Eq. (25) follows as

$$
\left(\kappa I_{\mathrm{b}}+\kappa I_{i}\right) \Delta V=I_{f, \phi+} \cdot A_{f, \phi+}+I_{f, \phi-} \cdot A_{f, \phi-}+\sum_{\text {otherfacesi }} I_{f, i} \cdot A_{f, i}
$$

where $\Delta V$ is the cell volume, $I_{f, \phi+}$ and $A_{f, \phi+}$ the face values of intensity and face area at wedge angle $+\Delta \omega / 2$ respectively, and similarly $I_{f, \phi-}$ and $A_{f, \phi+}$ at wedge angle $-\Delta \omega / 2$ as shown in Fig. 5 . In Eq. (26), only the two wedge face terms are isolated, since the others are not affected. Similar to the discussion on the specularly reflective boundary condition, the face values of intensity have to be consistent with the interpolation scheme used for other internal faces.

u

To illustrate the construction of the wedge boundary values, we use the following example, shown in Fig. 5 using step scheme. The ordinate direction $\hat{\boldsymbol{s}}_{i}$ points toward a positive azimuthal direction. Therefore, the lower wedge face (corresponding to $\Delta \phi=-\Delta \omega / 2$ ) needs a face value $I_{f, \phi-}=I_{C-}$ as if the face is internal, while the opposite wedge face does not require values, e.g., the face value is $I_{f, \phi+}=I_{C}$ in the discretized equation to be solved. In this case, the required $I_{C-}$ corresponds to $\Delta \phi=-\Delta \omega$, the wedge angle. Due to assigning $d \phi=-d \beta$ from Eqs. (24), (25), $\Delta \beta=-\Delta \phi=\Delta \omega$, leading to

$$
I_{f, \phi-}=I_{C-}=I\left(r, z,-\Delta \omega ; \theta_{i}, \beta_{i}\right)=\hat{I}\left(r, z ; \theta_{i}, \beta_{i}+\Delta \omega\right)
$$

where Eq. (17) is employed for the last equal sign. This equation gives the wedge face value for the ith ordinate using the cell-center intensity values of another ordinate with direction $\left(\theta_{i}, \beta_{i}+\Delta \omega\right)$.

On the other hand, if the ordinate direction points toward a negative azimuthal direction, the upper wedge face acquires its value from another ordinate as

$$
I_{f, \phi+}=I_{C+}=I\left(r, z,+\Delta \omega ; \theta_{i}, \beta_{i}\right)=\hat{I}\left(r, z ; \theta_{i}, \beta_{i}-\Delta \omega\right)
$$

while the lower wedge patch has $I_{f, \phi-}=I_{C}$

This construction shows that a conventional three-dimensional DOM solver can be used without modifications. The azimuthal derivative in the rotationally invariant formulation, Eq. (24), is 
implemented in a numerically consistent and stable fashion through the new boundary condition, which maps the scalar intensity of another specially chosen ordinate to the wedge boundaries. Furthermore, other boundary conditions are not affected, because the RTE implementation is unaffected.

\subsection{Similarities and differences between two boundary conditions}

The two boundary conditions share several identical features:

(1) Both boundary conditions are applied to wedge boundaries for axisymmetric problems using conventional three-dimensional DOM solvers, allowing the use of the same directional quadrature scheme, discretization procedure, and linear equation solver in solving both threeor two-dimensional cases.

(2) When the step scheme is used, boundary faces with outgoing ordinates require boundary face values. Those with incoming ordinates acquire their values from the cell centers after the cell center values are solved for.

(3) In both boundary conditions, when a boundary face requires an intensity value, the value is determined from the intensity values of another ordinate. Therefore, different ordinates are coupled and need an outer iteration to achieve a converged solution.

The two boundary conditions also have distinctions because of their different constructions:

(1) The specularly reflective boundary condition finds the intensity values from another ordinate according to Eq. (10). The azimuthal angle between the two ordinates varies with the ordinate direction, which could be as large as $180^{\circ}$ in case of normal incidence. The rotationally invariant boundary condition finds the intensity values from another ordinate with a fixed azimuthal separation of the wedge angle ( $\pm \Delta \omega$ ) according to Eqs. (27), (28).

(2) When the step scheme is used, the specularly reflective boundary condition copies the face intensity from another ordinate as Eq. (14), while rotationally invariant boundary condition copies cell center values as Eqs. (27), (28).

(3) The rotationally invariant boundary condition is limited to wedge geometry with a single azimuthal layer of cells. The specularly reflective boundary condition does not have this restriction. Aside from axisymmetric problems, it can be applied to periodic problems, such as discrete rotational symmetry and rectangular periodic geometries.

(4) The DOM RTE solver solves for the intensity in five dimensions with a specularly reflective boundary condition. The solution is not necessarily rotationally invariant. With the rotationally invariant boundary condition, the same DOM RTE solver solves for intensity in four dimensions with rotational invariance built-in.

\subsection{Directional interpolation}

Both newly proposed boundary conditions require finding intensity of another direction. The new direction is, in general, not within the set of ordinates unless the ordinate directions and the wedge open angle are chosen carefully. When the required direction is not resolved, an interpolation scheme 
is required to approximate the intensity of the unresolved direction using adjacent resolved ordinates, which depends on the details of the directional quadrature.

In this work, a simple equal partitioning is applied to both polar and azimuthal angles. The $\pi$ polar angles are equally divided into $N_{\theta}$ intervals, and the $2 \pi$ azimuthal angles are equally divided into $N_{\beta}$ intervals. Each ordinate corresponds to a polar and an azimuthal angle interval. The nominal direction of each ordinate is along the center of the polar and azimuthal angle interval covered. This division results in a total of $N_{\theta} \times N_{\beta}$ ordinates. When a nominal direction needed is different from any of the existing ordinates, its intensity is interpolated from the four ordinates that form a rectangular grid on the basis of polar and azimuthal angles. The situation is further simplified if the polar angle is measured from the axial direction, since only the azimuthal angle is changed in the new direction in both boundary conditions. As a result, interpolation is effectively only one-dimensional in the azimuthal angle.

\section{Results and discussions}

In this section sample calculations are carried out to demonstrate the effectiveness of the specularly reflective boundary condition and the rotationally invariant boundary condition for Discrete Ordinate Methods. The specularly reflective boundary condition may be applied to periodic or symmetry geometries. The consistency of the two boundary conditions is demonstrated within the first two examples. Then both boundary conditions are applied to the same one-dimensional radial radiation problem. The focus is the numerical accuracy and stability when directional interpolation is needed. Lastly, both boundary conditions are applied to wedge boundaries of an axisymmetric jet flame solved in a wedge geometry to investigate their accuracy and efficiency.

\subsection{Consistency of specularly reflective boundary conditions}

An axisymmetric radiation problem is considered here to demonstrate that the proposed specularly reflective boundary condition yields the identical results as that from a full geometry. A homogeneous emitting-absorbing medium is confined in an infinitely long cylindrical enclosure bounded by coldblack walls. The problem is one-dimensional in the radial direction, and has two symmetry conditions. One is along the azimuthal direction, the other one is along the axial direction. The problem therefore may be solved with four different geometries as shown in Fig. 6. Geometry A is a sufficiently long cylinder bounded by black-cold walls at both ends. The radial cross section at the center achieves the target solution asymptotically as the length of the cylinder increases. Specularly reflective boundary conditions, therefore, are not needed in this geometry. This geometry serves as a benchmark for the following consistency test. Geometry B has a wedge shape with one layer of cells along the azimuthal direction. It takes advantage of the axisymmetry of the problem. Both the additional front and back surfaces require the specularly reflective boundary condition. This geometry examines the consistency of specularly reflective boundary conditions in axisymmetric problems. Geometry $\mathrm{C}$ has a pie shape that has only one layer of cells along the axial direction. It utilizes axial periodicity, such that both top and bottom surfaces are specularly reflective. This geometry examines the consistency of the specularly reflective boundary condition in periodic problems. Geometry D utilizes both axial periodicity and axisymmetry. It has cells only along the radial direction. The specularly reflective boundary condition is applied to front, back, top and bottom surfaces. This geometry examines the 
consistency of specularly reflective boundary conditions in problems with combined rotational and periodic symmetries. Because the solution (e.g., incident radiation or radiative heat source) of this problem varies only radially, a successful boundary condition for the reduced geometries should produce identical results in all four geometries.

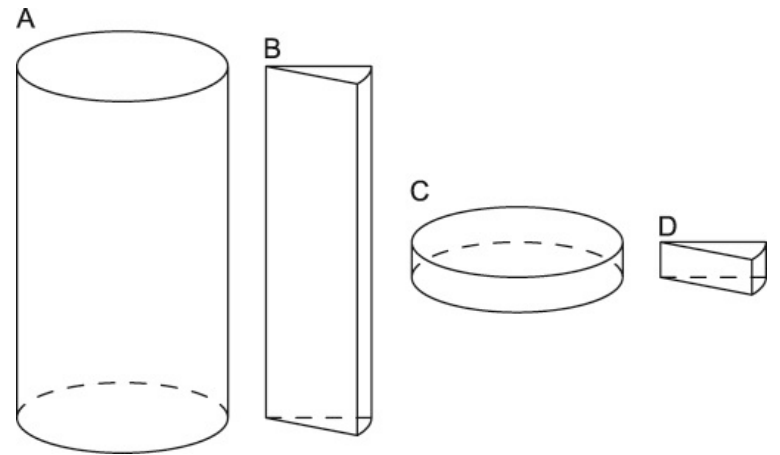

Fig. 6. Four geometries.

The same RTE implementation, discretization, directional quadrature scheme and linear equation solver are used in all four geometries, such that any deviations between geometries are due to the boundary condition only. A cylindrical grid with uniform spacing along axial, radial and azimuthal directions is used to discretize the RTE. The wedge geometries (Geometries B and D) have a $10^{\circ}$ opening angle. Geometries $A$ and $C$ have 36 azimuthal cells at $10^{\circ}$ each, correspondingly. Geometries $\mathrm{C}$ and $\mathrm{D}$ have identical height corresponding to the cell height in Geometries $\mathrm{A}$ and B. A total of 72 ordinates ( 2 polar and 36 azimuthal directions) are employed. This choice of a large number of azimuthal directions ensures that reflected ordinates are within the set of ordinates, which avoids complications from directional interpolations. The radial direction is discretized uniformly into 1000 cells. The radius of the cylinder is $1 \mathrm{~m}$, and the absorption coefficient is $1 \mathrm{~m}-1$. Emission is homogeneous.

The solutions of incident radiation normalized by the uniform emission $\left(G / 4 \pi I_{\mathrm{b}}\right)$ are compared in Fig. 7 against the exact answer. Results from all four geometries overlap with each other. This suggests the effectiveness of the proposed specular reflective boundary condition in dealing with both axisymmetric and periodic symmetries. The difference between the exact and the predicted results in all four cases is due to the fact that the polar direction has only two ordinates.

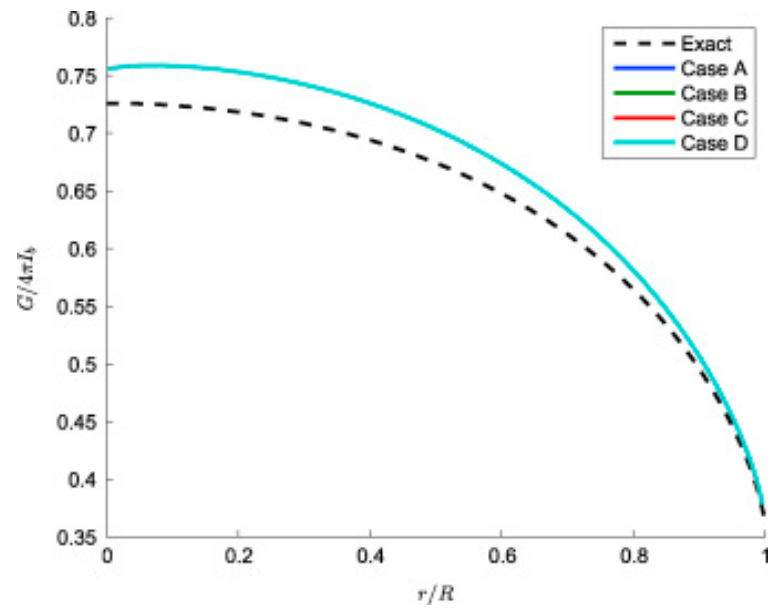


Fig. 7. Comparison of the normalized incident radiation predicted from four geometries.

\subsection{Consistency of rotationally invariant boundary conditions}

The consistency of rotationally invariant boundary condition is also examined in the same way as the specularly reflective boundary condition. Geometry A still serves as the benchmark, and is unchanged. Geometry $C$ is not needed, since it does not possess wedge boundaries. Geometries B and D are recalculated using the rotationally invariant boundary condition for the wedge face boundaries. For Geometry D, the up and bottom boundaries are specularly reflective.

As shown in Fig. 8, predictions from Geometries A, B and D overlap with others. The overlapped prediction between Geometries $A$ and $B$ suggests that the rotationally invariant boundary condition successfully reproduces the correct values on the wedge open boundaries. The prediction of Geometry $D$ overlaps with $B$ and $A$ suggests that the rotationally invariant boundary condition can be applied to problems involving other boundary conditions, although in principle the rotationally invariant formulation has a different radiative transfer equation formulation. This is due to the fact that the rotationally invariant boundary condition transforms a rotationally invariant RTE into a conventional three dimensional DOM RTE, so that other existing boundary conditions are still applicable.

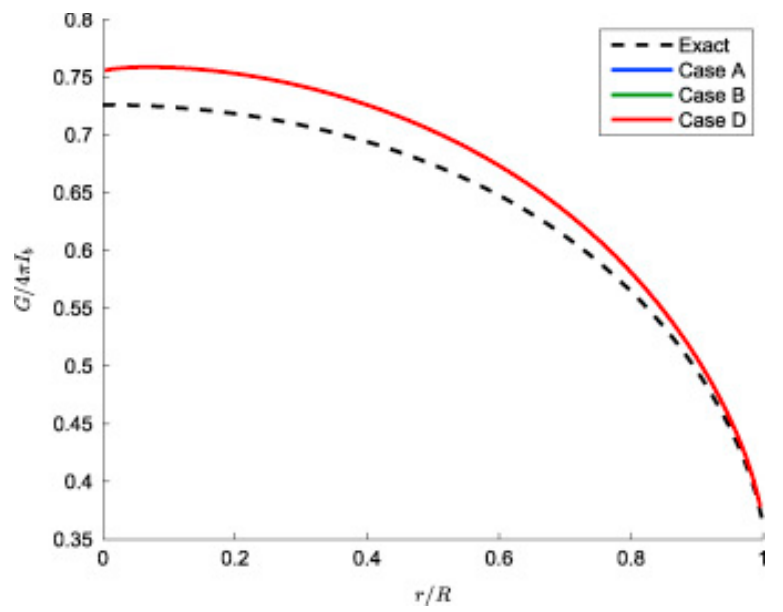

Fig. 8. Comparison of the normalized incident radiation predicted from different geometries.

\subsection{Comparisons for one-dimensional axisymmetric radiation with directional interpolation}

In practice, a small wedge angle is preferred (i.e., less than $10^{\circ}$ ) in order the retain numerical accuracy in approximating azimuthal derivatives as spatial derivatives between two wedge boundaries. Matching this small wedge angle in the azimuthal direction of ordinates requires a large number of ordinates to avoid azimuthal interpolations even if a few polar directions are employed. The resulting radiation simulation is computationally expensive without significant improvement in accuracy. When few azimuthal directions are employed, new directions needed by the aforementioned boundary conditions are no longer resolved by the ordinate systems. In this case, a directional interpolation is required to find the necessary intensity values from the resolved ordinates. Here we investigate the numerical accuracy of the boundary conditions when such directional interpolation is needed. 
The test case is the same one-dimensional radial radiation problem of the previous sections using geometry $D$ shown in Fig. 6 . Top and bottom boundaries are specularly reflective. Specularly reflected directions from these two boundaries are within the set ordinates in this study. Therefore, as shown in the previous sections, the accuracy and consistency are retained for these two boundaries because no directional interpolation is needed. Both new boundary conditions are then applied to the wedge boundaries. The comparisons in this section focuses on numerical performance with directional interpolation. For the specularly reflective boundary condition, this comparison is different from that in the previous section, in which azimuthal angles were chosen to avoid directional interpolations.

In Fig. 9, the radial profiles of normalized incident radiation predicted using the specularly reflective boundary condition on wedge boundaries are compared for different numbers of polar and azimuthal directions. The configurations are reported according to the number of polar directions multiplied by $(x)$ the number of azimuthal directions, which gives the total number of ordinates employed. The number of ordinates ranges from $2 \times 4$ for a coarse directional resolution to $16 \times 32$ for a fine resolution. All four ordinate configurations require directional interpolation on this $10^{\circ}$ wedge. When fewer number of ordinates are employed, e.g., $2 \times 4$, the solution deviates greatly from the exact solution. However, compared to Fig. 7, in which $2 \times 36$ ordinates were used without any directional interpolation, the under-prediction near the centerline (i.e., $r / R<0.2$ ) suggests that directional interpolation is numerically inaccurate for the specularly reflective boundary condition when too few ordinates are employed. As the number of ordinates increases, the solution improves. Especially, when $8 \times 16$ or more ordinates are employed, the solution is sufficiently close to the exact solution.



Fig. 9. Comparison of the normalized incident radiation predicted from four quadrature schemes using the specularly reflective boundary condition.

The same comparisons are made using the rotationally invariant boundary condition, as shown in Fig. 10. The numerical solution demonstrates consistent improvements with an increasing number of ordinates. The $4 \times 8$ configuration shows accuracy comparable to $P_{1}$. Further increasing the ordinates to $8 \times 16$ and beyond gives accurate predictions close to the exact solution. Also, the finest two directional resolutions give results identical to that using the specularly reflective boundary condition. 


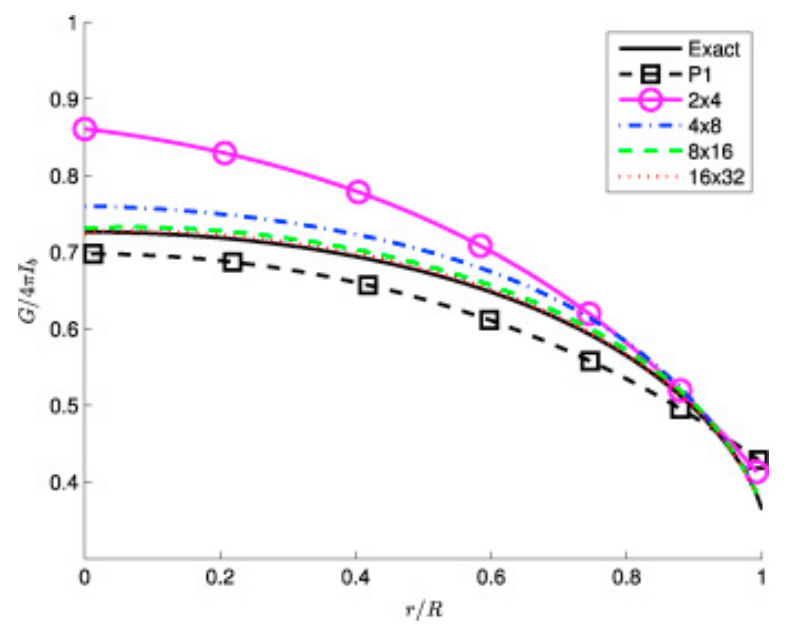

Fig. 10. Comparison of the normalized incident radiation predicted from four quadrature schemes using the rotationally invariant boundary condition.

\subsection{Axisymmetric jet flame}

The specularly reflective and rotationally invariant boundary conditions are further applied to an axisymmetric jet diffusion flame solved on a wedge mesh shown in Fig. 11. The flame was derived from the methane-air partially premixed Sandia Flame D [14] by artificially quadrupling the jet diameter [15], [16]. The wedge mesh has a $10^{\circ}$ opening angle. Previous solutions of time averaged profiles are used for the radiation calculation. Radial profiles of temperature and major species relevant to radiation at two axial locations are shown in Fig. 12. The axial location $1 \mathrm{~m}$ above the nozzle corresponds to significant CO production, while temperature peaks approximately at $1.4 \mathrm{~m}$ above the nozzle. The same flame was employed to investigate the accuracy and efficiency of $k$-distribution methods and $P_{N}$ RTE solvers [17]. In this section the results from Discrete Ordinate Methods are reported. The spectral model is the full-spectrum $k$-distribution method assembled from a narrowband $k$-distribution database [18]. This spectral model was known to give less than $2 \%$ error in the nongray spectral modeling of this flame [17]. Polar angles are measured from the axial direction ( $y$ axis shown in Fig. 11). Various numbers of polar and azimuthal ordinates are employed to investigate the accuracy. Three configurations are reported: they are $2 \times 4,4 \times 8$ and $8 \times 16$, respectively. Because the wedge angle is $10^{\circ}$, none of these configurations is an integer division of $360^{\circ}$ azimuthal angle, and therefore, directional interpolation is employed in all cases. 


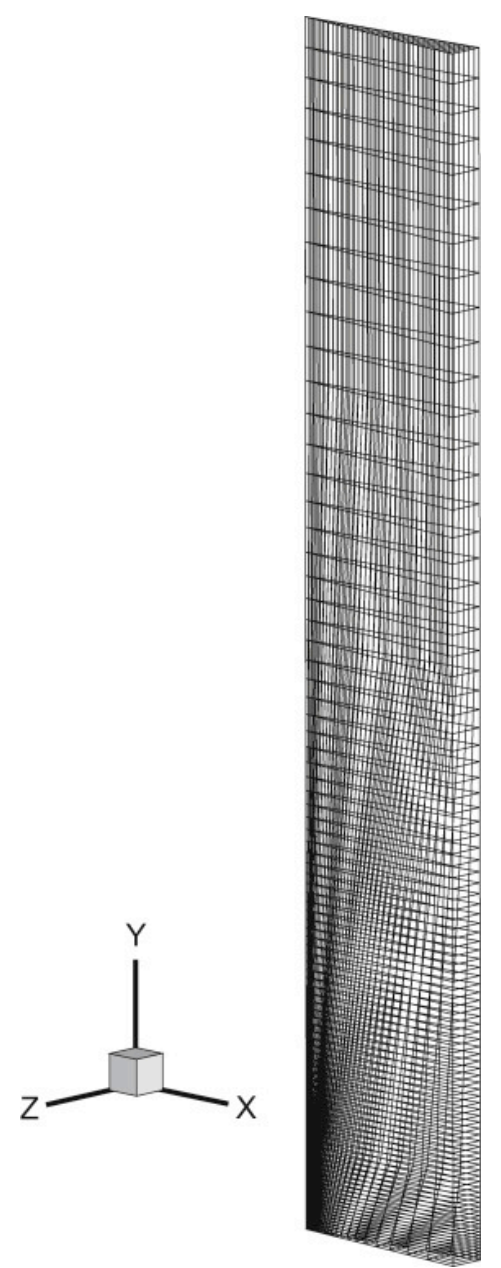

Fig. 11. Wedge shaped mesh was used to solve for flame profiles.

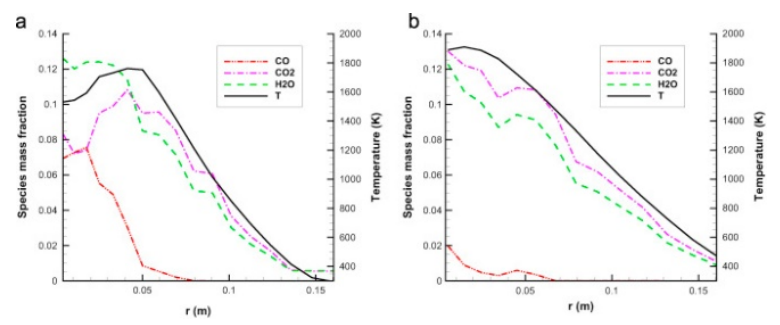

Fig. 12. Radial scalar profiles at $1 \mathrm{~m}$ above nozzle (left) and at $1.4 \mathrm{~m}$ above nozzle (right).

The radiative heat flux divergence $(\nabla \cdot \boldsymbol{q})$ or negative of radiative heat source in the energy equation predicted by different DOM configurations is compared at $1 \mathrm{~m}$ and $1.4 \mathrm{~m}$ above the nozzle in Fig. 13, Fig. 14, respectively. For reference, also shown in the figures are predictions from other radiation models and configurations, namely, the Optically Thin approximation (OT), line-by-line accurate Photon Monte Carlo, the full-spectrum $k$-distribution coupled with $P_{1}$ and the same fullspectrum $k$-distribution coupled with a full 3D DOM. Radiative emission as shown by the solid line with square markers is determined by the Optically Thin approximation (OT), which does not account for absorption. True radiative heat sources are determined by the Photon Monte Carlo method (PMC) coupled with a line-by-line spectral model, shown as dots with error bars. The error bar width is three times the standard deviation, which brackets the true radiative heat source with over $99 \%$ of 
confidence. Comparison between the PMC solution and the optically thin approximation (OT) suggest that this problem has significant amounts of self-absorption that requires an accurate RTE solver [17]. It has been reported that the absorption is entirely nongray in this gaseous flame, such that the gray spectral model fails to predict the absorption regardless of the performance of the RTE model [17]. Therefore, $P_{1}$ and DOM solvers hereafter all utilize the same full-spectrum $k$-distribution spectral model. The $P_{1}$ results are reproduced here to investigate the possibility of improvements from high order DOM solvers. Furthermore, the same spectral model is also coupled with the highest order DOM ( $8 \times 16$ here) in a three-dimensional cylindrical mesh, which does not involve the wedge boundary conditions introduced in this work. A successful wedge boundary condition should give consistent predictions approaching this 3D-DOM result as the number of ordinates increases.
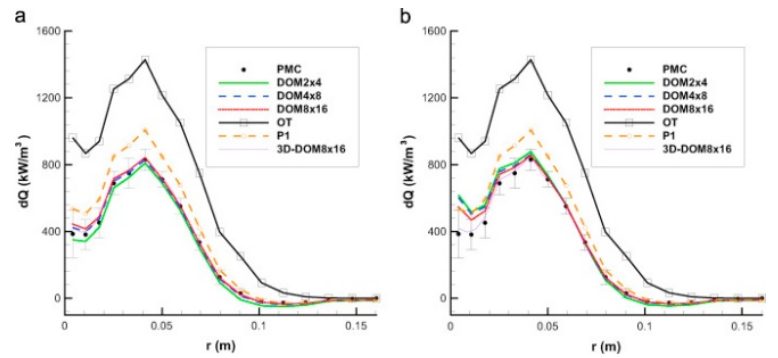

Fig. 13. Radial profiles of predictions of radiative heat flux divergence at $1 \mathrm{~m}$ above nozzle using rotational invariant boundary conditions (left) and specularly reflective boundary conditions (right) for the wedge surface.
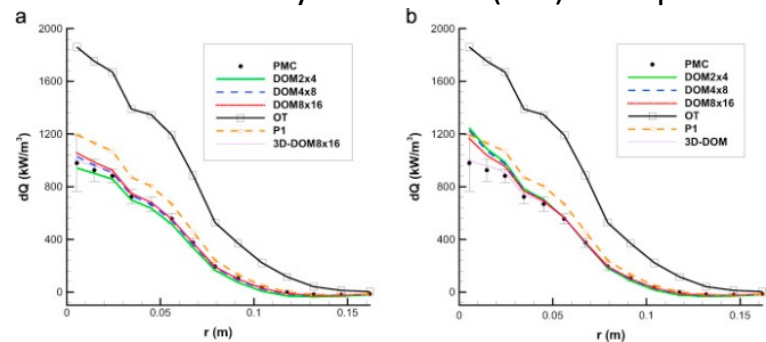

Fig. 14. Radial profiles of predictions of radiative heat flux divergence at $1.4 \mathrm{~m}$ above nozzle using rotational invariant boundary conditions (left) and specularly reflective boundary conditions (right) for the wedge surface.

Similar trends are found for the two boundary conditions at both downstream locations as shown in Fig. 13, Fig. 14. High-order three-dimensional DOM (labeled as 3D-DOM8×16) coupled with the fullspectrum $k$-distributions gives predictions very close to the PMC with a deviation far smaller than the uncertainty of the PMC. This suggests that this combination of the spectral model and DOM RTE is capable of providing highly accurate predictions.

Figs. 13 (a) and 14(a) show the DOM comparisons for when the rotationally invariant boundary condition is employed in the wedge geometry. The $2 \times 4$ configuration gives significant improvements over the $P_{1}$ RTE model. This configuration corresponds to one ordinate per octant, and is found to be an easy configuration to improve the RTE accuracy. Further increasing the number of ordinates gives little improvement because $2 \times 4$ is already close to the PMC.

When specularly reflective boundary condition is employed [Figs. 13(b) and 14(b)], improvements similar to the rotationally invariant boundary condition are also observed for intermediate and large radii, i.e., $r>0.04 m$. The accuracy drops near the centerline, because of numerical accuracy of the specularly reflective boundary condition drops when coupled with the directional interpolation for a 
small number of ordinates shown in the previous section. However, this does not invalidate the value of the specularly reflective boundary condition because it can achieve results consistent with its 3D counterpart when directional interpolation is not needed as shown in the previous sections.

Furthermore, it is applicable to other geometries with discrete rotational or periodic symmetry beyond the capability of the rotationally invariant boundary, especially when a carefully chosen directional quadrature scheme avoids the directional interpolation.

\subsection{Computational cost}

The computational cost of the previous flame examples averaged over all $k$-distribution quadrature points using two boundary conditions is shown in Table 1. If a small number of quadratures is used, the rotationally invariant boundary condition requires less CPU time. This time cost difference becomes smaller when the number of ordinates is increased. Since the rotationally invariant boundary condition always offers better accuracy, it is suggested in practical simulations.

Table 1. Total CPU time cost (s) for all 8 quadrature points of the full-spectrum $k$-distribution model.

\begin{tabular}{|l|l|l|l|}
\hline DOM configuration & $\mathbf{2 \times 4}$ & $\mathbf{4 \times 8}$ & $\mathbf{8 \times 1 6}$ \\
\hline Specularly reflective & 1.6 & 4.4 & 31.2 \\
\hline Rotationally invariant & 1 & 4.5 & 31.7 \\
\hline
\end{tabular}

\section{Conclusions}

In this work, two boundary conditions are proposed for numerical simulations of axisymmetric radiation problems using DOM on a wedge grid system. The specularly reflective boundary condition is similar to that employed in the Photon Monte Carlo method. It reduces computational geometry by exploring periodicity or symmetry of the problem. Expressing the boundary condition depends on the spatial discretization scheme of the gradient term. For the simplest step scheme, the specularly reflective boundary condition for ordinate intensity reduces to zero gradient (or fixed value) for incoming (or outgoing) directions relative to the boundary. Therefore, the intensity of outgoing ordinates at the boundary after reflection depends on the intensity of the incoming ordinates, but not vice versa. However, for higher order schemes the incoming and outgoing ordinates are fully coupled. Similarly, a rotationally invariant boundary condition is developed to enforce the rotational invariance properties possessed by the RTE but violated by conventional DOM directional discretization.

The consistency of the two boundary conditions is demonstrated with a one-dimensional radial problem in cylindrical coordinates that possesses both axisymmetry and plane symmetry. The numerical example of a jet flame solved on a wedge geometry, which possesses both radial and axial inhomogeneities, is also provided. The rotationally invariant boundary condition was found more accurate and efficient than the specularly reflective boundary condition in axisymmetric problems using wedge grids, when directional interpolation is needed to find the intensity of unresolved directions. 


\section{Acknowledgment}

The research conducted at University of California at Merced was sponsored by AFOSR under Grant number FA8650-15-C-2543. The research conducted at University of Wyoming was supported by a faculty start-up grant and Engineering Initiative funding for "Advanced Combustion Research Cluster". Several tests were conducted on ARCC supercomputers of University of Wyoming [19].

\section{References}

[1] M.F. Modest. Radiative heat transfer. (3rd ed.), Academic Press, New York (2013)

[2] P.J. Coelho. Advances in the discrete ordinates and finite volume methods for the solution of radiative heat transfer problems in participating media. J Quant Spectrosc Radiat Transf, 145 (2014), pp. 121-146

[3] E.H. Chui, G.D. Raithby, P.M.J. Hughes. Prediction of radiative transfer in cylindrical enclosures with the finite volume method. J Thermoph Heat Transf, 6 (4) (1992), pp. 605-611

[4] J.P. Moder, J.C. Chai, G. Parthasarathy, H.S. Lee, S.V. Patankar. Nonaxisymmetric radiative transfer in cylindrical enclosures. .Numer Heat Transf B, 30 (4) (1996), pp. 437-452

[5] M.Y. Kim. Assessment of the axisymmetric radiative heat transfer in a cylindrical enclosure with the finite volume method. Int J Heat Mass Transf, 51 (21-22) (2008), pp. 5144-5153

[6] Fiveland WA. A discrete ordinates method for predicting radiative heat transfer in axisymmetric enclosures. ASME Paper 82-HT-20; 1982.

[7] S.W. Baek, M.Y. Kim. Modification of the discrete-ordinates method in an axisymmetric cylindrical geometry. Numer Heat Transf B, 31 (3) (1997), pp. 313-326

[8] J.Y. Murthy, S.R. Mathur. Radiative heat transfer in axisymmetric geometries using an unstructured finite-volume method. Numer Heat Transf B, 33 (4) (1998), pp. 397-416

[9] J. Liu, H.M. Shang, Y.S. Chen. Development of an unstructured radiation model applicable for twodimensional planar, axisymmetric, and three-dimensional geometries. J Quant Spectrosc Radiat Transf, 66 (1) (2000), pp. 17-33

[10] M.B. Salah, F. Askri, K. Slimi, S.B. Nasrallah. Numerical resolution of the radiative transfer equation in a cylindrical enclosure with the finite-volume method. Int J Heat Mass Transf, 47 (2004), pp. 2501-2509

[11] W. Tian, W.K.S. Chiu. A two-dimensional scheme for axisymmetric radiative heat transfer using the finite-volume method. Numer Heat Transf Part B: Fundam, 47 (3) (2005), pp. 199-211

[12] S.R. Mathur, J.Y. Murthy. Radiative heat transfer in periodic geometries using a finite volume scheme. J Heat Transf, 121 (2) (1999), pp. 357-364

[13] P. Kumar, V. Eswaran. A methodology to solve 2D and axisymmetric radiative transfer problems using a general 3D solver. J Heat Transf, 12 (2013), p. 124501

[14] R.S. Barlow, J.H. Frank. Effects of turbulence on species mass fractions in methane/air jet flames. Proc Combust Inst, 27 (1998), pp. 1087-1095

[15] G. Li, M.F. Modest. Importance of turbulence-radiation interactions in turbulent diffusion jet flames. .J Heat Transf, 125 (2003), pp. 831-838

[16] A. Wang, M.F. Modest, D.C. Haworth, L. Wang. Monte Carlo simulation of radiative heat transfer and turbulence interactions in methane/air jet flames. J Quant Spectrosc Radiat Transf, 109 (2) (2008), pp. 269-279 
[17]

J. Cai, R. Marquez, M.F. Modest

Comparisons of radiative heat transfer calculations in a jet diffusion flame using spherical harmonics and k-distributions

J Heat Transf, 136 (11) (2014), p. 112702

[18]

J. Cai, M.F. Modest

Improved full-spectrum k-distribution implementation for inhomogeneous media using a narrowband database

J Quant Spectrosc Radiat Transf, 141 (2014), pp. 65-72

[19]

Advanced Research Computing Center, Mount Moran: IBM System X cluster. Laramie, WY: University of Wyoming, URL. 〈http://n2t.net/ark:/85786/m4159c〉; 2012. 